\title{
Kinematic age determinations of planetary nebula central stars
}

\author{
Thaise S. Rodrigues and Walter J. Maciel \\ Astronomy Department, University of São Paulo \\ Rua do Matão 1226, 05508-090 São Paulo SP, Brazil \\ email: tsrodrigues@usp.br - maciel@astro.iag.usp.br
}

\begin{abstract}
Central stars of planetary nebulae (CSPN) have a relatively large mass interval, so that it is expected that these stars also have different ages, typically above 1 Gyr. Apart from the properties of the CSPN themselves, the problem of age determination is also important in the context of the chemical evolution of the Galaxy, for instance in the understanding of the time variation of chemical abundance gradients. In this work, we estimated the ages of a sample of CSPN on the basis of some correlations between their kinematic properties and the expected ages. According to these correlations, the observed dispersions in the $U, V, W$ velocities are uniquely defined by the stellar ages. The adopted correlations were derived from the recent Geneva-Copenhagen survey of galactic stars. Preliminary results suggest the most CSPN in the galactic disk have ages under 3 Gyr. These results are also compared with some recent age distributions based on independent correlations involving the nebular chemical abundances.
\end{abstract}

Keywords. planetary nebulae: general, stars: AGB and post-AGB

\section{Introduction}

Planetary nebulae $(\mathrm{PN})$ are ejected from low and intermediate mass stars, with about 0.8 to 8 solar masses on the main sequence, so that it is expected that their central stars $(\mathrm{CSPN})$ may have a large age range, typically above 1 Gyr. The age determination is a complex problem and there are no unique and reliable methods. However, in the context of the chemical evolution of the Galaxy, it is important to determine the ages of CSPN, for instance in the study of time variations of the chemical abundance gradients (cf. Maciel et al. 2003, 2005). In this work, we estimated CSPN ages based on correlations between kinematic properties and the ages. We considered two samples: (i) the same used by our group in a previous work (Maciel et al. 2010) which contains 234 well studied PN; (ii) a more complete sample based on the catalogue by Durand et al. (1998) which contains $867 \mathrm{PN}$ with accurate radial velocities.

\section{Kinematic age distribution}

The age determination of CSPN from kinematic properties is based on the age-velocity dispersion relations that show a smooth increase in the dispersion of the $U, V, W$ components of the spatial velocity with age. We used a relation estimated from the GenevaCopenhagen survey (Holmberg et al. 2009), and developed two methods to estimate the CSPN velocity dispersions.

\subsection{Method 1}

With the heliocentric distance, galactic coordinates and Local Standard of Rest radial velocity of the PN, we derived the rotation velocity $\left(\theta_{R}\right)$ of the CSPN at a given galactocentric distance assuming circular orbits. We adopted $R_{0}=8 \mathrm{kpc}$ and $\theta_{0}=220 \mathrm{~km}$ 
$\mathrm{s}^{-1}$ for the galactocentric distance and the rotation velocity of the Sun. Considering a galactic rotation curve $\left(\theta_{c}\right)$, we assumed that the diference between the rotation velocity of the CSPN and the rotation curve at a given galactocentric distance $\left(\Delta \theta=\left|\theta_{R}-\theta_{c}\right|\right)$ is proportional to the velocity dispersion. We have used the following: (i) two distance scales - a short scale, by Maciel (1984) and a long scale, by Stanghellini et al. (2008) - to ensure that the individual distance uncertainties would not affect the derived age distribution; (ii) the radial velocities from the catalogue by Durand et al. (1998); (iii) two galactic rotation curves - Maciel \& Lago (2005), derived from PN, and Clemens (1985) derived from $\mathrm{H}$ II and $\mathrm{CO}$ regions. We considered two possibilities: $\Delta \theta$ proportional to the dispersion of the $\mathrm{V}$ component and to the total spatial velocity. We have then derived 8 different age distributions.

\subsection{Method 2}

We calculated the $U, V, W$ components using the relations by Boesgaard \& Tripicco (1986). We made three assumptions concerning the tangential velocities and proper motions of the CSPN: (i) we assumed that, in average, the tangential velocities are equal to the radial velocities; (ii) since CSPN are distant objects, as first approximation, we neglected the proper motions; (iii) alternatively, we considered nonzero values for the proper motions in right ascension $\left(\mu_{\alpha}\right)$ and declination $\left(\mu_{\delta}\right)$ assuming that $\left|\mu_{\alpha}\right|=\left|\mu_{\delta}\right|$. We calculated the velocity dispersions and the total velocity. We have then derived 16 age distributions using both distance scales mentioned in sect. 2.1. Concerning assumption (iii), the proper motions could have different signs, so we derived the age distributions for every combination of signs and found that they do not change significantly.

\section{Results}

According to both methods, most of the CSPN have ages under 5 Gyr, peaking at 1-2 Gyr, and the fraction of CSPN under 3 Gyr is about $50-70 \%$ for Method 1 and $70-90 \%$ for Method 2. The age distributions are apparently not affected by the uncertainties of the distances, since the histograms for both adopted distance scales are similar. The main uncertainty in the ages is due the adopted age-velocity dispersion. The age uncertainty is of approximately 25\%, which is around 1.2 Gyr for most objects, for both methods and samples. Our results are similar to those by Maciel et al. (2010), which were obtained from three methods based on abundance correlations; however, our age distributions indicate somewhat lower ages for most CSPN in our samples. (FAPESP/CNPq)

\section{References}

Boesgaard, A. M., \& Tripicco, M. J., 1986, ApJ, 303, 724

Clemens, D. P., 1985, ApJ, 295, 422

Durand, S., Acker, A., \& Zijlstra, A., 1998, A\& $A S, 132,13$

Holmberg, J., Nordström, B., \& Andersen, J., 2009, Aछ A, 501, 941

Maciel, W. J., 1984, A\&AS, 55, 253

Maciel, W. J., Costa, R. D. D., \& Idiart, T. E. P., 2010, $A \& A$, 512, A19

Maciel, W. J., Costa, R. D. D., \& Uchida, M. M. M., 2003, A\& A, 397, 667

Maciel, W. J. \& Lago, L. G., 2005, Rev. Mexicana A\&SA, 41, 383

Maciel, W. J., Lago, L. G., \& Costa, R. D. D., 2005, A\&A, 433, 127

Stanghellini, L., Shaw, R. A., \& Villaver, E., 2008, ApJ, 689, 194 